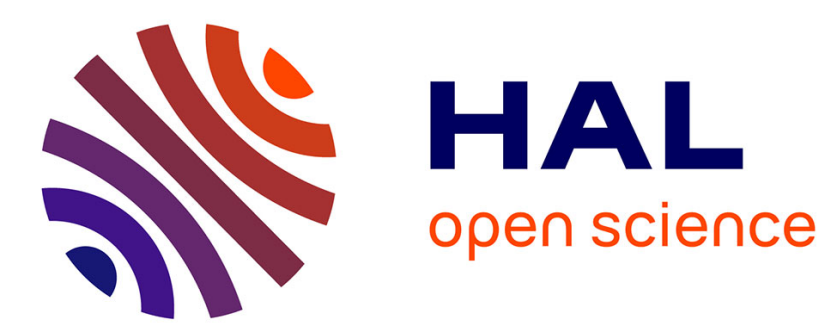

\title{
Aggregation in a sheared 2D dispersion of spheres with attractive interactions
}

\author{
C. Camoin, R. Blanc
}

\section{To cite this version:}

C. Camoin, R. Blanc. Aggregation in a sheared 2D dispersion of spheres with attractive interactions. Journal de Physique Lettres, 1985, 46 (2), pp.67-74. 10.1051/jphyslet:0198500460206700 . jpa00232479

\section{HAL Id: jpa-00232479 https://hal.science/jpa-00232479}

Submitted on 1 Jan 1985

HAL is a multi-disciplinary open access archive for the deposit and dissemination of scientific research documents, whether they are published or not. The documents may come from teaching and research institutions in France or abroad, or from public or private research centers.
L'archive ouverte pluridisciplinaire HAL, est destinée au dépôt et à la diffusion de documents scientifiques de niveau recherche, publiés ou non, émanant des établissements d'enseignement et de recherche français ou étrangers, des laboratoires publics ou privés. 
Classification

Physics Abstracts

$05.40-47.90$

\title{
Aggregation in a sheared 2D dispersion of spheres with attractive interactions
}

\author{
C. Camoin and R. Blanc \\ Département de Physique des Systèmes Désordonnés (*), \\ Université de Provence, Centre de St-Jérôme, 13397 Marseille Cedex 13, France
}

(Reçu le 10 septembre 1984, accepté le 29 novembre 1984)

\begin{abstract}
Résumé. - Nous présentons une expérience d'agrégation dans une suspension bidimensionnelle de particules sphériques macroscopiques soumises à un cisaillement. L'agrégation est due à des forces interfaciales attractives dont on peut faire varier l'intensité. On introduit une quantité sans dimension $\boldsymbol{A}$ traduisant l'importance relative des forces attractives et de l'effet du cisaillement. On observe diverses configurations liées à la valeur de $A$. En particulier, dans le cas où les amas présentent une structure fractale, on trouve une dimension d'Hausdorff à deux dimensions égales à 1,7 $\pm 0,05$.

Abstract. - We present aggregation experiments in a bidimensional suspension of macroscopic spherical particles undergoing a shear. The aggregation is due to attractive capillary forces the value of which can be changed. We introduce a dimensionless quantity $A$ expressing the relative importance of capillary forces and of the shear effect. We observe various aggregate configurations related to the values of $A$. In particular when the clusters show a fractal structure we find one Hausdorff dimension equal to $1.7 \pm 0.05$.
\end{abstract}

\section{Introduction.}

The formation of aggregates in a system of particles is a very common phenomenon observed in various experimental situations such as, for instance, the flocculation in colloids [1], the coagulation of aerosols [2], and free radical chemical gelation [3]. When the clusters grow from particles initially dispersed in a fluid. numerous basic physical phenomena come into action in this nonequilibrium growth process : translational and rotational Brownian motion. attractive and/or repulsive forces between particles. shear of the fluid and hydrodynamic interactions are some of the most important.

In this Letter, we report the first experimental results obtained from the study of aggregates in a two-dimensional dispersion of macroscopic spheres submitted to a shear when attractive forces between particles are present.

The first stage in the clustering process is the formation of a doublet of particles which was first studied by Smoluchowski [4]. He examined the simple case of a particle moving by translational Brownian diffusion. meeting a " target " particle and sticking onto it in an irreversible

$\left(^{*}\right)$ Equipe de Recherche Associée au C.N.R.S., no 1000. 
way when the two particles come in contact. In such a treatment. one neglects the hydrodynamic interactions between particles and in particular the slow elimination of the film of fluid between close particles (lubrication) [5]. One also neglects the interparticle forces (described for instance. in the DLVO theory [1]). Depending upon their variation with interparticle distances, these forces give rise to an irreversible aggregation of the two particles coming into contact or to the formation of a doublet in which the two particles remain separated by a small distance. In this last case. the doublet may eventually be broken due to various causes such as Brownian motion or hydrodynamic shear. The formation of a doublet may also have a convective aspect related to the existence of a shear in the fluid. In this case. one has a convective-diffusive phenomenon the effect of which is to increase the rate of formation of doublets [6]. At the limit where the particles are large enough (size typically larger than $1 \mu \mathrm{m}$ ) for the Brownian and non-hydrodynamic effects to be unimportant, one observes the formation of a temporary reversible doublet. whose mean life is inversely proportional to the shear rate [7].

The growth of clusters containing a larger number of particles has been the object of numerous recent studies in two limiting cases (purely Brownian or purely hydrodynamic).

1) In the Brownian case. studies on diffusion-limited aggregation have been initiated by Witten and Sander [8]. They study the growth of a cluster by progressive adjunction of particles reaching it after a random walk and irreversibly sticking to it. The spatial structure of these clusters has a scale invariance and one can define a Hausdorff dimension $D_{F}$ related to the Euclidean dimension $d$ by the approximate relation $D_{\mathrm{F}} / d \sim 0.83$ for $2<d<5\left(D_{\mathrm{F}}=1.68\right.$ for $\left.d=2\right)$ [9]. In the same spirit. the recent works by Kolb et al. [10]. Meakin [11]. Vicksek and Family [12] deal with the kinetical growth of clusters by diffusion-limited aggregation of isolated particles and clusters.

2) In the purely hydrodynamic case, a percolation-like approach has been proposed by de Gennes [13]. It is based on the existence of reversible temporary doublets whose life time, $\theta$, varies with the shear rate $G\left(\theta \sim G^{-1}\right)$. The rate of formation of such a doublet is proportional to $G$. Thus the probability $P$ for one object to be involved at any time in a collision doublet is independent of $G$ and depends only on the density of particles. This behaviour is different from that of hard spheres in a gas for which the contacts are instantaneous (the probability $P$ approaches zero). When the particle density increases, the probability for a sphere to be involved in a larger structure increases and multiplets are formed. These clusters are dynamical in the sense that they are permanently gaining and losing particles: so at any time a well defined size distribution of clusters is observed. The existence and the size distribution of these clusters have been experimentally established in the case of bidimensional dispersions of macroscopic monodisperse spheres [14]. Using a dynamic molecular-like simulation of dispersions of 25 to 100 particles. Bossis and Brady [15] have studied the formation and the geometrical structure of such clusters.

\section{Experimental procedure.}

The suspensions used in this work consist of spherical solid particles made of synthetic material (Hostalen PP, diameter $d=3.17 \mathrm{~mm}$ ) neutrally buoyant in a viscous fluid layer. The fluid layer thickness $h$ varies from $d$ to $0.3 d$ so that attractive forces (described below in more detail) can act between spheres (no attraction is observed when $h=d$ ). The viscous fluid layer floats on water. A cross section perpendicular to the surface (represented in Fig. 1) shows that spheres are not in contact with water. The dispersion can be regarded as bidimensional because the centres of the spheres lie in the same horizontal plane as long as the superficial density $\phi_{\mathrm{s}},\left(\phi_{\mathrm{s}}=\right.$ $N_{0} \pi d^{2} / 4 s$ with $N_{0}=$ number of sphere. $d=$ diameter of spheres, $s=$ area of the viscometer gap) is not too high. In the experiments reported in this Letter, we have used a single concentration $\phi_{\mathrm{s}}=0.15$. The dispersion is placed in a Couette viscometer $\left(R_{1}=\right.$ inner radius $=6 \mathrm{~cm}$ : 


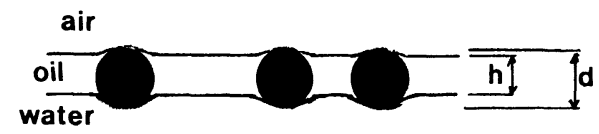

Fig. 1. - View in a vertical section of the suspension showing the spheres coated with oil.

$R_{2}=$ outer radius $=18 \mathrm{~cm}$ ) whose inner cylinder rotates with angular speed. $\Omega$. Three rotation speeds have been used $\left(0.21 \mathrm{~s}^{-1}, 0.16 \mathrm{~s}^{-1}, 0.105 \mathrm{~s}^{-1}\right)$. Under these conditions, the particle Reynolds number remains lower than 0.1 . The depth of water $(\sim 15 \mathrm{~cm})$ under the dispersion is sufficient that we can neglect the effect of the solid bottom of the viscometer on the flow in the viscous layer.

When $\Omega=0$ (dispersion at rest) and $h<d$. one observes that two neighbouring spheres attract each other and come in contact. Figure 1 shows that the viscous fluid-air and viscous fluid-water interfaces are deformed by the spheres so that the area of the two interfaces increases and the gravitational potential energy is modified. As far as we know, there is no theoretical treatment of such a system. We can however refer to the work of Marcerou [17] who examined the case of two vertical cylinders partially immersed in water. The free energy minimization of such a system (taking into account the gravitational and the interfacial energy) shows that the resultant interaction is attractive. In our experimental situation it also appears that the interaction between spheres is always attractive. Under the action of these forces, two spheres approach each other according to a complex dynamics resulting from the hydrodynamical Stokes forces and lubrication effects. These latter effects can induce a very strong slowing down of the rate of approach of the spheres when they are very near each other and appear optically to be in contact.

When $\Omega \neq 0$ (sheared suspension) and $h=d$ (attractive forces equal to 0 ), two particles on two close circular trajectories can form a temporary doublet in a perfect reversible way if the Reynolds number is very small, e.g. when inertial effects are negligible with regard to viscous ones. Thus the shear allows the spheres to approach first and later to separate.

If one adds to these shear effects the attractive forces observed when $h<d$, perfect reversibility no longer exists : two spheres drawn near each other by the shear will exert on each other an attraction: it acts first with the shear then opposite to it when the shear tends to separate the spheres. When these forces are intense or the shear too weak (Ref. [13b] introduces the notion of a shear threshold below which separation is impossible), one can observe the formation of a permanent doublet. In the case where the spheres separate again. the effect of attraction decreases the difference between the radii of the circular trajectories of the two spheres and increases the capture probability in a later encounter.

When one no longer considers a pair of spheres but a dispersion with a finite concentration, phenomena are more complex. However the mechanisms described in the case of two spheres allow us to understand the cluster formation and the reversibility (or lack of it) which depends on the existence and strength of attractive forces. The use of a cylindrical Couette apparatus produces a continuous flow so that the observation time can be much larger than the growth time of the clusters. In our experiments a given observation was initiated from a random particle distribution with a given angular speed and a given layer thickness and made when the system had reached a stationary state. The time necessary to attain the final state is a function of experimental conditions. For instance if $A$ (see its definition below) is about one, this time is two or three hours. 


\section{Experimental results.}

3. 1 HYDRODYNAMIC AND CAPILLARY FORCES. - In order to quantify our experimental results, we must introduce a quantity expressing the competition between hydrodynamic forces due to the shear which brings particles together and separates them and the forces of surface tension which tend to aggregate them.

The hydrodynamic force effect can be measured by a mean shear rate $\langle G\rangle$

$$
\langle G\rangle=\frac{1}{R_{2}-R_{1}} \int_{R_{1}}^{R_{2}} G(r) \mathrm{d} r
$$

where $G(r)$ is the shear rate at $\mathrm{M}$ with $\mathrm{OM}=r\left(R_{2}>r>R_{1}, \mathrm{O}\right.$ being the centre of the cylinders). Owing to the values of $R_{1}$ and $R_{2},\langle G\rangle$ is about $\Omega / 2$.

We characterize the strength of the attractive forces between spheres by the time $\tau$ necessary for two particles placed at a given distance, $l$, to come into visual contact in the suspension at rest [18]. The value of $l(l=1 \mathrm{~cm})$ has been chosen by reference to the capillary length (a few millimeters). Figure 2 shows the variations of $\tau$ versus $h / d$ for two different viscous fluids.

A dimensionless number taking into account the relative importance of shear and attractive effects can be defined as

$$
A=\langle G\rangle \cdot \tau \text {. }
$$

It compares the time of attraction $\tau$ and the mean lifetime of reversible doublets $G^{-1}$. Thus, operating with different angular rotation speeds and with various thicknesses of the layer of viscous fluids, we can explore a large range of values of $A$. A given value of $A$ may be obtained with several pairs of values of $\langle G\rangle$ and $\tau$.

3.2 RESULTS AND INTERPRETATION. - Our observations are summarized in the following table.

\begin{tabular}{|c|c|l|}
\hline Fig. 3 & $A$ & Characterization \\
\hline a & 0 or $\rightarrow 0$ & Branched clusters \\
\hline b & $5 \times 10^{-2}$ to $5 \times 10^{-1}$ & Unique cluster showing no crystallization \\
\hline c & 1 to 4 & Crystallized clusters in finite number \\
\hline d & 8 to 10 & Statistical clusters with annular structures \\
\hline e & $\rightarrow \infty$ & Statistical clusters \\
\hline
\end{tabular}

Case a (cf. Fig. 3a). - When $A$ is equal to zero or small (no rotation or intense capillary forces associated with a low shear) the clusters show branched structures (Fig. 3a) with voids of different scales.

In figure 4 we have plotted $\log N$ versus $\log R$ where, for a given cluster, $N$ represents the number of centres of spheres inside a circle of radius $R$ (normalized with regard to the particle radius). The slope of the linear best fit of experimental points gives the Hausdorff dimension $D_{\mathrm{F}}=1.7 \pm 0.05$. This value is in good agreement with the one obtained in the numerical simulation of Witten and Sander [8] and the one $(1.64 \pm 0.13)$ derived from the results obtained in a similar experiment by Allain and Jouhier [16] where the two-dimensional aggregation was 


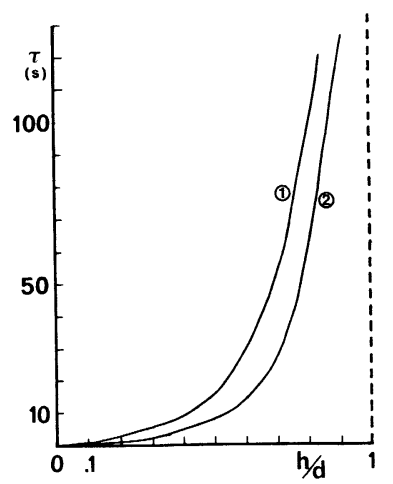

Fig. 2. - Variation of the time contact $\tau$ versus $h / d$ (where $h$ is the thickness of oil layer and $d$ the sphere diameter). Curves 1 and 2 correspond to two kinds of fluids.

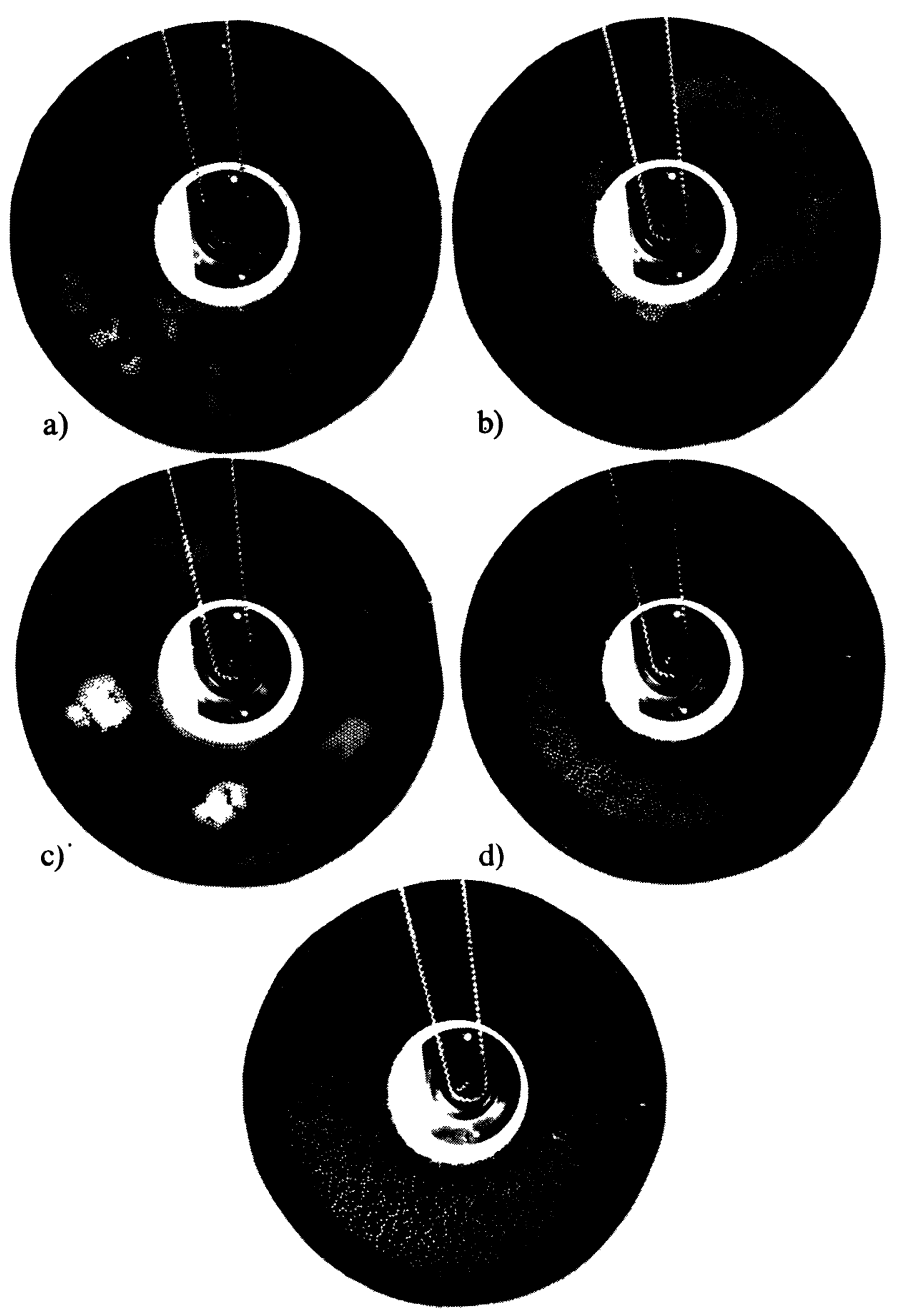

e)

Fig. 3. - Aspects of the dispersion for a) $A$ about 0: b) $A \sim 0.1:$ c) $A \sim 1:$ d) $A \sim 5:$ e) $A \rightarrow \infty$. 
also obtained with capillary attractive forces and negligible shear. We must point out that the latter value is obtained from the relation between the mean radius of a cluster and the number of objects in it [19].

However our results are in good agreement with the model of Witten and Sander, this accord can be fortuitous because from observation we deduce that we have a cluster-cluster aggregation process rather than a particle-cluster one.

Case $b$ (Fig. 3b). - For $A$ of a few tenths, we observe a single cluster containing all the spheres (Fig. $3 b$ ). We can distinguish three parts in this assembly : the inner one entirely surrounds the inner cylinder and rotates rigidly with the cylinder speed. In the outer part, there is a bounded solid-like structure rotating with a smaller angular velocity: in the intermediate part, where there is an important shear, the particle motions are intense.

In this case, and in the previous one (case a), clusters of spheres are formed under attractive force effects and cannot be disconnected by hydrodynamic ones. The shear rate is probably below the dissociation threshold introduced in reference [13b]. In both cases, the largest clusters grow by aggregation of smaller ones obtained in the first stages of the process. While, in case a, attractive forces between clusters give rise to a blocked structure which cannot be reorganized by the weak shear, such a rearrangement occurs in case $b$ leading to a denser structure.

Case $c$ (Fig. 3c). - For $A$ of the order of one, particles are grouped in a finite number of clusters (from 3 to 8) distributed in the central part of the gap and showing a hexagonal structure with several point defects (vacancies). During and after their formation these clusters rotate, thus creating a secondary flow around them : when a smaller cluster approaches a bigger one, it rotates in an opposite sense to the one expected due to the shear. Such a secondary flow may help to stabilize the cluster structures and lead to the final situation shown in figure 3c. For this value of $A$, we are still in the irreversible case but there is now a complementarity between the two effects : attractive and hydrodynamic forces bring spheres into contact and the shear structures the clusters to make them compact (hexagonal structure). Furthermore, the number of clusters increases with the shear rate. Similar behaviour has been observed in suspensions of alumina particles where the mean size of clusters decreases when the shear increases [20].

Case $d$ (Fig. 3d). - For $A$ varying from approximately 4 or 5 to 10, clusters lose their hexagonal structure and tend to form an annular ring located in the central part of the viscometer gap (Fig. 3d). In this case, the observed clusters are not permanent and can gain and lose particles. The shear rate is probably higher than the shear threshold and the attractive forces should increase the life time of a given cluster. This point remains to be quantitatively checked. One can see that almost all spheres are located in the middle part of the gap. Such a behaviour with depleted layers has been observed, for instance, in hemorheology (plasmatic layers) [21]. In our case, we can assume that such a phenomenon is due to the fact that the resultant attractive forces acting on the spheres located near the inner or outer cylinders are directed towards the middle of the gap.

Case $e$ (Fig. 3e). - For higher values of $A$ (no attractive interaction) we can observe statistical clusters covering the whole viscometer gap area. These perfectly reversible clusters have been studied in detail previously [15] (Fig. 3e).

\section{Conclusion.}

The experiments described in this Letter show that the introduction of attractive forces in a dispersion of macroscopic spheres produce important modifications in the geometrical organization of clusters. The relative strength of these forces and hydrodynamic forces due to the shear seems to govern the aggregation processes in our experimental situation. These variations 


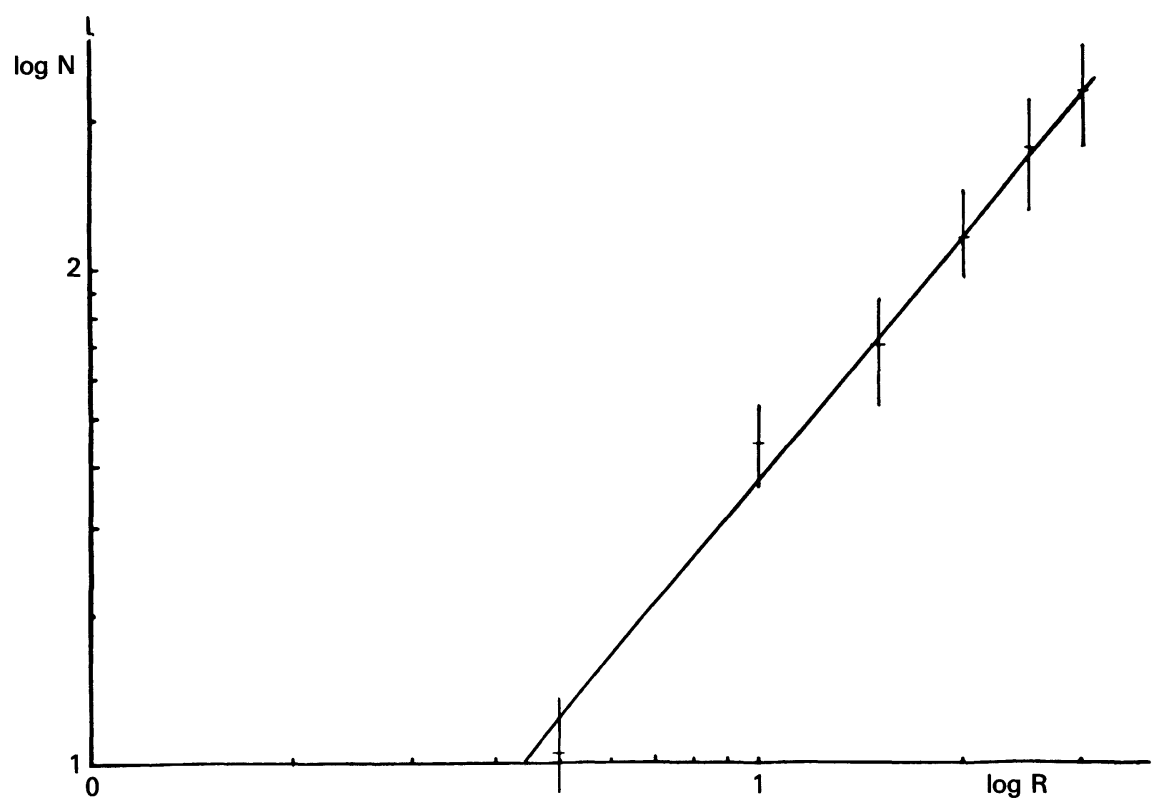

Fig. 4. - $\log N$ versus $\log R . N=$ number of centres of spheres inside a circle of radius $D . R=D / a$ where $a$ is the sphere radius (error bars result from measurements realized on 50 different configurations).

allow us to observe a wide variety of geometrical configurations, from self-similar objects, analogous to the clusters obtained in numerical simulations of the diffusion-limited aggregation, to statistical and reversible clusters analogous to percolation clusters. Between these two limiting configurations the joint effects of the attractive and hydrodynamic forces lead to a regular and completely different organization of the dispersion. The role of topological constraints, more important in two- than in three-dimensions, should also be considered even if, as shown by experimental observations on three-dimensional suspensions [20], it is not a crucial one. Supplemented by a more precise study of attractive forces, these experiments may give access to the study of some important questions such as :

- the kinetic aspects in aggregation processes : the constitution and evolution of clusters from individual spheres and, on a larger scale, the constitution and evolution of big clusters from smaller ones,

- the influence of aggregation on the rheological properties of the dispersions,

- the role of the concentration : from the point of view of the final geometrical configuration and from that of the rheological properties.

\section{Acknowledgments.}

We thank E. Guyon for stimulating and helpful discussions during this work. 


\section{References}

[1] a) Hiemenz, P. C., Principles of Colloid and Surface Chemistry (Dekker, ed.) 1977.

b) Lyklema, J., in Colloides et interfaces (Editions de Physique, Les Ulis, France) 1984, p. 3.

[2] Friedlander, S. K., Smoke, Dust and Haze (J. Wiley, New York) 1977.

[3] a) Jouhier, B., Allain, C., Gauthier-Manuel, B., Guyon, E., " Percolation, structure and processes ", Annals Israel Phys. Soc. 5 (1983) 167.

b) Stauffer, D., Coniglio, A., Adam, M., Adv. Polym. Sci. 44 (1982) 103.

[4] Smoluchowski, M. V., Z. Phys. Chem. 92 (1977) 129.

[5] LANDAU et LiFCHITZ, Mécanique des Fluides (Editions MIR) 1971.

[6] VAN DE Ven, T. and Mason, S. G.,

a) Colloid Polym. Sci. 255 (1977) 794.

b) J. Colloid Interface Sci. 57 (1976) 505.

c) Colloid Polym. Sci. 255 (1977) 468.

[7] Bartok, W. and Mason, S. G., J. Colloid Sci. 12 (1957) 243.

[8] WitTen, T. A. Jr. and SANDER, L. M.,

a) Phys. Rev. Lett. 47 (1981) 1400.

b) Phys. Rev. B 27 (1983) 5686.

[9] Meakin, P., Phys. Rev. A 27 (1983) 604.

[10] Kolb, M., Bottet, R., Jullien, R., Phys. Rev. Lett. 51 (1983) 1123.

[11] Meakin, P., Phys. Rev. Lett. 51 (1983) 1119.

[12] VickseK, T. and Family, F., Phys. Rev. Lett. 52 (1984) 1669.

[13] De Gennes, P. G.,

a) J. Physique 40 (1979) 783.

b) Physico Chemical Hydrodynamics III (1981) p. 231.

[14] a) Belzons, M., Blanc, R., Boulllot, J. L., Camoin, C., C.R. Hebd. Séan. Acad. Sci., Pairis 292 (1981) 939.

b) Bouillot, J. L., Camoin, C., Belzons, M., Blanc, R., Guyon, E., Adv. Colloid Interface Sci. 17 (1982) 299.

c) Blanc, R., Belzons, M., Camoin, C., Boulllot, J. L., Rheol. Acta 22 (1983) 505.

[15] a) Bossis, G. and Brady, J. F., J. Chem. Phys. 80 (1984) 5141.

b) Brady, J. F. and Bossis, G., Dynamic simulation of sheared suspension : rheology, to appear in Proc. IX Int.,Congr. Rheol.

[16] Allain, C. and Jouhier, B., J. Physique Lett. 44 (1983) L-421.

[17] Marcerou, J. P., Thèse de Doctorat d'Etat, Université de Bordeaux (1982).

[18] A direct measurement of the strength of these forces versus the interparticle distance is now in progress.

[19] According to the usual notations (cf. Ref. [8]), the dimensionality thus calculated is $D_{\alpha}$ whereas in our case we determine $D_{\beta}$. These two quantities are generally regarded as equivalent.

[20] Bourrier, P. and ChapUis, J., private communication.

[21] Quemada, D., J. Physique Colloq. 37 (1976) C1-9. 